\title{
Pre-Hausdorff and Hausdorff objects in the category of quantale-valued closure spaces
}

\author{
Muhammad Qasim ${ }^{1}$ (D), Bin Pang*2 (D) \\ ${ }^{1}$ Department of Mathematics, School of Natural Sciences (SNS), National University of Sciences and \\ Technology (NUST), H-12 Islamabad, 44000, Pakistan \\ ${ }^{2}$ School of Mathematics and Statistics, Beijing Institute of Technology, 100081 Beijing, PR China
}

\begin{abstract}
In previous papers, several $T_{0}$ and Hausdorff objects in topological categories are introduced and compared. The main objectives of this paper are to characterize $\overline{T_{0}}, T_{0}, T_{1}$ and pre- $\overline{T_{2}}$ objects in the category of quantale-valued closure space as well as to examine their mutual relationship.
\end{abstract}

Mathematics Subject Classification (2020). 54A05, 54B30, 54D10, 54A40, 18F60

Keywords. $\mathcal{V}$-closure space, $\mathcal{V}$-topological space, topological category, separation, Hausdorff objects

\section{Introduction}

It is an established fact that closure operators play a vital role not only in mathematics including algebra [34], logic [18], calculus [29] and topology [14,23], but also in physics such as quantum logic and representation theory of physical systems [2,3]. In 1940, G. Birkhoff [13] found the relations between the collection of closed sets of a closure space and complete lattice. Afterwards, their interrelations have emerged as the issues of major concerns for mathematicians [16]. Moreover, G. Aumann [4] investigated the closure structures on contact relations which have applications in social sciences.

In 1991, Baran [5] introduced $T_{0}$ and $T_{1}$ objects in a set-based topological category by using generic element method [21] which is further elucidated in topos theory. Also, he introduced pre-Hausdorff objects in an arbitrary topological category which are reduced to pre-Hausdorff topological space $(X, \tau)$, i.e., for each distinct point $x, y \in X$, if the set $\{x, y\}$ is not an indiscrete space, then the points $x$ and $y$ have disjoint neighborhoods [10]. The most important use of pre-Hausdorff objects is to define various forms of Hausdorff objects [11], $T_{3}$ and $T_{4}$ objects [8], regular, completely regular and normal objects [9] in arbitrary topological categories. In 1994, M. V. Mielke [27] showed that the pre-Hausdorff objects play an important role in general theory of geometric realization. Later, M.V. Mielke [28] showed that pre-Hausdorff objects are important tools for the characterization of the decidable objects in topos theory.

\footnotetext{
*Corresponding Author.

Email addresses: muhammad.qasim@sns.nust.edu.pk, qasim99956@gmail.com (M. Qasim), pangbin1205@163.com (B. Pang)

Received: 21.05.2020; Accepted: 23.09.2020
} 
With the development of fuzzy set theory, many mathematical structures have been equipped with fuzzy sets, such as fuzzy topology [24,39], fuzzy convexity [32,33], fuzzy convergence $[30,31,40]$ and so on. Considering the fuzzy counterparts of closure structures, it has been generalized by introducing some suitable quantales on closure structures [24,25]. This motivates us to consider separation properties of the topological category of quantalevalued closure spaces.

The main objectives of this paper are stated as follows:

(i) to characterize $\overline{T_{0}}, T_{0}$ and $T_{1}$ in the category of quantale-valued closure space,

(ii) to provide the characterization of pre-Hausdorff and several forms of Hausdorff objects in quantale-valued closure space,

(iii) to examine how these separation axioms are related.

\section{Preliminaries}

In this paper, let $\mathcal{V}=(V, \otimes, k)$ be a (unital, but not necessarily commutative) quantale, i.e., a complete lattice with a monoid structure whose binary operation $\otimes$ satisfies the following properties: for all $\alpha_{i}, \beta \in V,\left(\bigvee_{i \in I} \alpha_{i}\right) \otimes \beta=\bigvee_{i \in I}\left(\alpha_{i} \otimes \beta\right)$ and $\beta \otimes\left(\bigvee_{i \in I} \alpha_{i}\right)=$ $\bigvee_{i \in I}\left(\beta \otimes \alpha_{i}\right)$, where $k$ is an identity (neutral) element.

A quantale $(V, \otimes, k)$ is called an integral quantale if $k=\top$.

In a quantale $(V, \otimes, k)$, if $p \in V$ and $p \neq \top$, then $p$ is called the prime element if $\alpha \wedge \beta \leq p$ implies $\alpha \leq p$ or $\beta \leq p$ for all $\alpha, \beta \in V$.

Let $X$ be a nonempty set, $P X$ denotes the power set of $X$ and $V^{X}$ denotes the set of all maps from $X$ to $\mathcal{V}$.

Definition 2.1 (cf. [25]). A $\mathcal{V}$-valued closure structure on set $X$ is a map $c: P X \longrightarrow \mathcal{V}^{X}$ satisfying

(i) $\forall x \in A \subseteq X: k \leq(c A)(x)$ (Reflexivity),

(ii) $\forall A, B \subseteq X, x \in X:\left(\bigwedge_{y \in B}(c A)(y)\right) \otimes(c B)(x) \leq(c A)(x)$ (Transitivity).

The pair $(X, c)$ is called a $\mathcal{V}$-valued closure space.

Definition 2.2 (cf. [25]). A $\mathcal{V}$-valued topological structure on set $X$ is a map $c: P X \longrightarrow$ $\nu^{X}$ satisfying

(i) $c$ is a $\mathcal{V}$-valued closure structure on $X$,

(ii) $\forall x \in X$ and $\emptyset$, the empty set: $(c \emptyset)(x)=\perp$,

(iii) $\forall x \in X$ and $\forall A, B \subseteq X: c(A \cup B)(x)=(c A)(x) \vee(c B)(x)$.

The pair $(X, c)$ is called a $\mathcal{V}$-valued topological space.

A map $f:(X, c) \longrightarrow(Y, d)$ is called continuous (or contractive) if $(c A)(x) \leq d(f A)(f x)$ for all $A \subseteq X$ and $x \in X$.

Let V-Cls (resp. V-Top) denote the category with $\mathcal{V}$-valued closure spaces (resp. $\mathcal{V}$-valued topological spaces) as objects and contractive maps as morphisms. Note that V-Top is the full subcategory of V-Cls.

Remark 2.3 (cf. [25]). A $\mathcal{V}$-valued closure structure $c$ on $X$ satisfies the monotonicity condition, i.e., $\emptyset \neq B \subseteq A \subseteq X \Longrightarrow c B \leq c A$. Furthermore, if $\mathcal{V}$ is an integral quantale or $c$ is finitely additive, then the restriction $B \neq \emptyset$ is not needed.

Example 2.4. (i) For terminal quantale 1, 1-Cls $=1$-Top $\cong$ Set [25].

(ii) Consider $\mathcal{V}=(2, \wedge, \top)$, where $2=\{\perp<\top\}$. Then 2-Cls $\cong$ Cls and 2-Top $\cong$ Top [25], where Cls is the category of closure spaces and continuous maps [15] and Top is the category of topological spaces and continuous maps.

(iii) If quantale $\mathcal{V}=(([0, \infty], \geq),+, 0)$ (Lawvere's quantale) [17], then $\mathcal{V}$-Top $\cong$ App, where App is the category of approach spaces and contraction maps [26]. Moreover, we have $\mathcal{V}$-Cls $\cong \mathbf{C l s}^{\prime}$, where $\mathbf{C l s}^{\prime}$ is the category considered in [37]. 
(iv) Consider the quantale $\triangle_{\&}=(\triangle, \otimes, k)$ of all distance distribution functions $\varphi$ : $[0, \infty] \longrightarrow[0,1]$ that satisfy $\varphi(\beta)=\sup _{\alpha<\beta} \varphi(\alpha)$ for all $\beta \in[0, \infty]$ with $(\varphi \otimes \xi)(\gamma)=$ $\sup \varphi(\alpha) \& \xi(\beta)$, where $\&$ is Lukasiewicz operation on $[0,1]$ defined by $\alpha \& \beta=$ $\max \{\alpha+\beta-1,0\}$. The $\otimes$-neutral function $k$ satisfies $k(0)=0$ and $k(\alpha)=1$ for all $\alpha>0$. Then, $\triangle_{\&}$-Top $\cong$ ProbApp $_{\&}[24,25]$ of probabilistic approach spaces defined in [19].

A functor $\mathcal{U}: \mathcal{E} \longrightarrow$ Set (the category of sets and functions) is called topological if $(i)$ $\mathcal{U}$ is concrete (i.e., faithful and amnestic), (ii) $\mathcal{U}$ consists of small fibers and (iii) every $\mathcal{U}$-source has a unique initial lift or equivalently, each $\mathcal{U}$-sink has a unique final lift $[1,35]$.

Note that a topological functor which has a left adjoint is called the discrete functor.

Lemma 2.5 (cf. [25]). Let $\mathcal{V}$ be a quantale, $\left(X_{i}, c_{i}\right)$ be a collection of $\mathcal{V}$-valued closure spaces and $\left(f_{i}: X \longrightarrow\left(X_{i}, c_{i}\right)\right)_{i \in I}$ be a source. Then, for all $x \in X$ and $A \subseteq X$,

$$
(c A)(x)=\bigwedge_{i \in I} c_{i}\left(f_{i} A\right)\left(f_{i} x\right)
$$

is the initial structure on $X$.

Lemma 2.6 (cf. [25]). Let $X$ be a non-empty set and $(X, c)$ be a $\mathcal{V}$-valued closure space.

(i) The discrete $\mathcal{V}$-valued closure structure on $X$ is given by

$$
\forall x \in X, \forall A \subseteq X,\left(c_{d i s} A\right)(x)= \begin{cases}k, & x \in A, \\ \perp, & x \notin A .\end{cases}
$$

(ii) The indiscrete $\mathcal{V}$-valued closure structure on $X$ is given by $\left(c_{\text {ind }} A\right)(x)=\top$.

Note that for a quantale $\mathcal{V}$, the category V-Cls is a topological category over Set [25].

\section{3. $T_{0}$ and $T_{1}$ Quantale-valued closure spaces}

Let $X$ be a non-empty set, $X^{2}=X \times X$ and the wedge $X^{2} \vee_{\triangle} X^{2}$ be two disjoint copies of $X^{2}$ identified along with the diagonal. In other words, $X^{2} \vee \triangle X^{2}$ is the pushout of $\triangle: X \longrightarrow X^{2}$ along itself. More precisely, if $i_{1}$ and $i_{2}: X^{2} \longrightarrow X^{2} \vee \triangle X^{2}$ denote the inclusion of $X^{2}$ as the first and second factor, respectively, then $i_{1} \triangle=i_{2} \triangle$ is the pushout diagram [5].

A point $(x, y)$ in $X^{2} \vee_{\triangle} X^{2}$ is denoted by $(x, y)_{1}$ (resp. $\left.(x, y)_{2}\right)$ if it is in the first (resp. second) component. Note that $(x, y)_{1}=(x, y)_{2}$ iff $x=y$.

Definition 3.1 (cf. [5]). A map $A: X^{2} \vee \triangle X^{2} \longrightarrow X^{3}$ is called a principal axis map provided that

$$
A(x, y)_{i}= \begin{cases}(x, y, x), & i=1 \\ (x, x, y), & i=2 .\end{cases}
$$

Definition 3.2 (cf. [5]). A map $S: X^{2} \vee \triangle X^{2} \longrightarrow X^{3}$ is called a skewed axis map provided that

$$
S(x, y)_{i}= \begin{cases}(x, y, y), & i=1 \\ (x, x, y), & i=2 .\end{cases}
$$

Definition 3.3 (cf. [5]). A map $\nabla: X^{2} \vee_{\triangle} X^{2} \longrightarrow X^{2}$ is called a folding map provided that $\nabla(x, y)_{i}=(x, y)$ for $i=1,2$. 
Definition 3.4. Let $U: \mathcal{E} \longrightarrow$ Set be a topological functor and $X \in O b(\mathcal{E})$ with $U(X)=$ $B$.

(i) $X$ is called $\overline{T_{0}}$ provided that the initial lift of the $U$-source $\left\{A: B^{2} \vee \triangle B^{2} \longrightarrow\right.$ $U\left(X^{3}\right)=B^{3}$ and $\left.\nabla: B^{2} \vee_{\triangle} B^{2} \longrightarrow U D\left(B^{2}\right)=B^{2}\right\}$ is discrete, where $D$ is the discrete functor [5].

(ii) $X$ is called $T_{0}$ provided that $X$ doesn't contain an indiscrete subspace with at least two points [38].

(iii) $X$ is called $T_{1}$ provided that the initial lift of the $U$-source $\left\{S: B^{2} \vee \triangle B^{2} \longrightarrow\right.$ $U\left(X^{3}\right)=B^{3}$ and $\left.\nabla: B^{2} \vee_{\triangle} B^{2} \longrightarrow U D\left(B^{2}\right)=B^{2}\right\}$ is discrete [5].

Remark 3.5. In Top (the category of topological spaces and continuous maps), $\overline{T_{0}}$ and $T_{0}$ (resp. $T_{1}$ ) are reduced to the following statement: For each $x, y \in X$ with $x \neq y$, there exists a neighborhood of $x$ which doesn't contain $y$ or (resp. and) there exists a neighborhood of $y$ which doesn't contain $x$ [7].

Theorem 3.6. Let $(X, c)$ be a $\mathcal{V}$-valued closure space. $(X, c)$ is $\overline{T_{0}}$ if and only if for all $x, y \in X$ with $x \neq y$, there exist $B \subseteq X$ with $x \in B, y \notin B$ and $C \subseteq X$ with $y \in C, x \notin C$ such that $c(B)(y) \wedge c(C)(x) \wedge k=\perp$, where $k$ is the tensor-neutral element.

Proof. Suppose $(X, c)$ is $\overline{T_{0}}$. For all $x, y \in X$ with $x \neq y$, let $\left\{(x, y)_{1}\right\} \subseteq D \subseteq X^{2} \vee_{\triangle} X^{2}$ and $(x, y)_{2} \in X^{2} \vee \triangle X^{2}$. Note that

$$
\begin{gathered}
c_{d i s}(\nabla D)\left(\nabla(x, y)_{2}\right)=c_{d i s}(\nabla D)(x, y)=k, \\
k \leq c\left(\pi_{1} A D\right)\left(\pi_{1} A(x, y)_{2}\right)=c\left(\pi_{1} A D\right)(x),
\end{gathered}
$$

since $x \in \pi_{1} A D$,

$$
c(C)(x)=c\left(\pi_{2} A D\right)\left(\pi_{2} A(x, y)_{2}\right)=c\left(\pi_{2} A D\right)(x)
$$

and

$$
c(B)(y)=c\left(\pi_{3} A D\right)\left(\pi_{3} A(x, y)_{2}\right)=c\left(\pi_{3} A D\right)(y) .
$$

Since $(x, y)_{2} \notin\left\{(x, y)_{1}\right\}$ and $(X, c)$ is $\overline{T_{0}}$, by Lemma 2.5 ,

$$
\begin{gathered}
\perp=\bigwedge\left\{c_{d i s}(\nabla D)\left(\nabla(x, y)_{2}\right), c\left(\pi_{1} A D\right)\left(\pi_{1} A(x, y)_{2}\right),\right. \\
\left.c\left(\pi_{2} A D\right)\left(\pi_{2} A(x, y)_{2}\right), c\left(\pi_{3} A D\right)\left(\pi_{3} A(x, y)_{2}\right)\right\} \\
=\bigwedge\{k, c(B)(y), c(C)(x)\},
\end{gathered}
$$

and consequently, $\bigwedge\{k, c(B)(y), c(C)(x)\}=\perp$.

Conversely, let $\bar{c}$ be an initial structure on the wedge $X^{2} \vee \triangle X^{2}$ induced by $A: X^{2} \vee_{\triangle}$ $X^{2} \longrightarrow U\left(X^{3}, c^{3}\right)=X^{3}$ and $\nabla: X^{2} \vee \triangle X^{2} \longrightarrow U\left(X^{2}, c_{d i s}\right)=X^{2}$, where $c^{3}$ is the product $\mathcal{V}$-valued closure structure on $X^{3}, c_{\text {dis }}$ is the discrete $\mathcal{V}$-valued closure structure on $X^{2} \vee \triangle X^{2}$ and $\pi_{j}: X^{3} \rightarrow X$ is the projection map for $j=1,2,3$.

Suppose $u \in X^{2} \vee_{\triangle} X^{2}$ and $D$ is a non-empty subset of $X^{2} \vee_{\triangle} X^{2}$.

Case I: If $\nabla u=(x, x) \in \nabla D$ for some $x \in X$, then $u=(x, x)_{1}$ or $u=(x, x)_{2} \in D$, and it follows that $\bar{c}(D)(u)=k$, where $k$ is the tensor neutral element.

Case II: If $\nabla u=(x, x) \notin \nabla D$, then $c_{d i s}(\nabla D)(\nabla u)=\perp$ since $c_{d i s}$ is the discrete $\mathcal{V}$-valued closure structure and consequently, $\bar{c}(D)(u)=\perp$.

Case III: Suppose $\nabla u=(x, y)$ for some $x, y \in X$ with $x \neq y$ and it follows that $u=(x, y)_{i}, i=1,2$.

(i) If $u=(x, y)_{1},(x, y)_{2} \in D$, then $\nabla u \in \nabla D$ and $\pi_{j} A u \in \pi_{j} A D$ for $j=1,2,3$, and consequently, $\bar{c}(D)(u)=k$.

(ii) If $u \notin D$, then $\nabla u=(x, y) \notin \nabla D$, and it follows that

$$
c_{\text {dis }}(\nabla D)(\nabla u)=c_{\text {dis }}(\nabla D)(x, y)=\perp,
$$

and consequently, $\bar{c}(D)(u)=\perp$. 
(iii) Suppose that $u=(x, y)_{1} \notin D$ but $(x, y)_{2} \in D$. It follows that

$$
c_{d i s}(\nabla D)\left(\nabla(x, y)_{1}\right)=c_{\text {dis }}(\nabla D)(x, y)=k
$$

and

$$
k \leq c\left(\pi_{1} A D\right)\left(\pi_{1} A(x, y)_{1}\right)=c\left(\pi_{1} A D\right)(x) .
$$

Since $x \in \pi_{1} A D$, we have

$$
c(B)(y)=c\left(\pi_{2} A D\right)\left(\pi_{2} A(x, y)_{1}\right)=c\left(\pi_{2} A D\right)(y)
$$

and

$$
c(C)(x)=c\left(\pi_{3} A D\right)\left(\pi_{3} A(x, y)_{1}\right)=c\left(\pi_{3} A D\right)(x) .
$$

By Lemma 2.5, it follows that

$$
\begin{aligned}
\bar{c}(D)(u)= & \bigwedge\left\{c_{\text {dis }}(\nabla D)\left(\nabla(x, y)_{1}\right), c\left(\pi_{1} A D\right)\left(\pi_{1} A(x, y)_{1}\right),\right. \\
\left.c\left(\pi_{2} A D\right)\left(\pi_{2} A(x, y)_{1}\right), c\left(\pi_{3} A D\right)\left(\pi_{3} A(x, y)_{1}\right)\right\} & \\
= & \bigwedge\{k, c(B)(y), c(C)(x)\}=\perp .
\end{aligned}
$$

By the assumption that $\bigwedge\{k, c(B)(y), c(C)(x)\}=\perp$.

(iv) Similarly, if $u=(x, y)_{2} \notin D$ but $(x, y)_{1} \in D$, it follows that $\bar{c}(D)(u)=\perp$.

Hence, for all $u \in X^{2} \vee \triangle X^{2}$ and all non-empty subset $D$ of $X^{2} \vee \triangle X^{2}$, we have

$$
\bar{c}(D)(u)= \begin{cases}k, & u \in D \\ \perp, & u \notin D .\end{cases}
$$

By Lemma $2.6(\mathrm{i}), \bar{c}$ is the discrete $\mathcal{V}$-valued closure structure on $X^{2} \vee \triangle X^{2}$. Thus, $(X, c)$ is $\overline{T_{0}}$.

Corollary 3.7. Let $(X, c)$ be a $\mathcal{V}$-valued closure space, where $\mathcal{V}$ is an integral quantale and $\mathcal{V}$ has a prime bottom element. $(X, c)$ is $\overline{T_{0}}$ if and only if for all $x, y \in X$ with $x \neq y$, there exist $B \subseteq X$ with $x \in B, y \notin B$ and $C \subseteq X$ with $y \in C, x \notin C$ such that $c(B)(y)=\perp$ or $c(C)(x)=\perp$.

Proof. It follows from Theorem 3.6 and definitions of prime bottom elements and integral quantales.

Theorem 3.8. Let $(X, c)$ be a $\mathcal{V}$-valued closure space. $(X, c)$ is $T_{0}$ if and only if for all $x, y \in X$ with $x \neq y, c(\{x\})(y)<\top$ or $c(\{y\})(x)<\top$.

Proof. Suppose $(X, c)$ is $T_{0}$. Let $D=\{x, y\}$ and $c_{D}$ be the initial $\mathcal{V}$-valued closure structure induced by $i: D \longrightarrow(X, c)$. For all $x, y \in X$ with $x \neq y, c_{D}(\{x\})(y)=$ $c(i\{x\})(i(y))=c(\{x\})(y)$ or $c(\{y\})(x)=c(i\{y\})(i(x))=c(\{y\})(x)$. By Lemma 2.6 (ii), it follows that $c(\{x\})(y)<\top$ or $c(\{y\})(x)<\top$. Otherwise, $c(\{x\})(y)=\top=c(\{y\})(x)$, and $X$ contains an indiscrete subspace with at least two elements.

Conversely, let for all $x, y \in X$ with $x \neq y, c(\{x\})(y)<\top$ or $c(\{y\})(x)<\top$. Suppose $D$ is an indiscrete subspace of $X$ with at least two elements and $x, y \in D$ with $x \neq y$. Let $c_{D}$ be the initial $\mathcal{V}$-valued closure structure induced by $i: D \longrightarrow(X, c)$. It follows immediately that $\top=c_{D}(\{x\})(y)=c(i\{x\})(i(y))=c_{D}(\{x\})(y)$ and $\top=c_{D}(\{y\})(x)=$ $c(i\{y\})(i(x))=c_{D}(\{y\})(x)$, and consequently, $c(\{x\})(y)=\top=c(\{y\})(x)$, a contradiction to our assumption. Therefore, $X$ doesn't contain an indiscrete subspace with at least two elements. Hence, by Definition 3.4 (ii), $(X, c)$ is $T_{0}$.

Theorem 3.9. Let $(X, c)$ be a $\mathcal{V}$-valued closure space. $(X, c)$ is $T_{1}$ if and only if for all $x, y \in X$ with $x \neq y$, there exist $B \subseteq X$ with $x \in B, y \notin B$ and $C \subseteq X$ with $y \in C, x \notin C$ such that $c(B)(y) \wedge k=\perp=c(C)(x) \wedge k$, where $k$ is the tensor-neutral element. 
Proof. Suppose $(X, c)$ is $T_{1}$. For all $x, y \in X$ with $x \neq y$, let $\left\{(x, y)_{1}\right\} \subseteq D \subseteq X^{2} \vee_{\triangle} X^{2}$ and $(x, y)_{2} \in X^{2} \vee \triangle X^{2}$. Note that

since $x \in \pi_{1} S D$,

$$
\begin{gathered}
c_{d i s}(\nabla D)\left(\nabla(x, y)_{2}\right)=c_{d i s}(\nabla D)(x, y)=k, \\
k \leq c\left(\pi_{1} S D\right)\left(\pi_{1} S(x, y)_{2}\right)=c\left(\pi_{1} S D\right)(x),
\end{gathered}
$$

and

$$
c(C)(x)=c\left(\pi_{2} S D\right)\left(\pi_{2} S(x, y)_{2}\right)=c\left(\pi_{2} S D\right)(x)
$$

$$
k \leq c\left(\pi_{3} S D\right)\left(\pi_{3} S(x, y)_{2}\right)=c\left(\pi_{3} S D\right)(y)
$$

since $y \in \pi_{3} S D$. By the assumption that $(X, c)$ is $T_{1}$ and by Lemma 2.5 ,

$$
\begin{aligned}
\perp= & \bigwedge\left\{c_{d i s}(\nabla D)\left(\nabla(x, y)_{2}\right), c\left(\pi_{1} S D\right)\left(\pi_{1} S(x, y)_{2}\right),\right. \\
& \left.c\left(\pi_{2} S D\right)\left(\pi_{2} S(x, y)_{2}\right), c\left(\pi_{3} S D\right)\left(\pi_{3} S(x, y)_{2}\right)\right\} \\
= & \bigwedge\{k, c(C)(x)\},
\end{aligned}
$$

and consequently, $c(C)(x) \wedge k=\perp$.

Similarly, if $\left\{(x, y)_{2}\right\} \subseteq D \subseteq X^{2} \vee_{\triangle} X^{2}$ and $(x, y)_{1} \in X^{2} \vee_{\triangle} X^{2}$, then

$$
\begin{aligned}
\perp & \left.=\bigwedge\left\{c_{\text {dis }}(\nabla D)\left(\nabla(x, y)_{1}\right), c\left(\pi_{j} S D\right\}\right)\left(\pi_{j} S(x, y)_{1}\right), j=1,2,3\right\} \\
& =\bigwedge\{k, c(B)(y)\},
\end{aligned}
$$

and consequently, $c(B)(y) \wedge k=\perp$.

Conversely, let $\bar{c}$ be an initial structure on the wedge $X^{2} \vee_{\triangle} X^{2}$ induced by $S: X^{2} \vee \triangle$ $X^{2} \longrightarrow U\left(X^{3}, c^{3}\right)=X^{3}$ and $\nabla: X^{2} \vee_{\triangle} X^{2} \longrightarrow U\left(X^{2}, c_{d i s}\right)=X^{2}$, where $c^{3}$ is the product $\mathcal{V}$-valued closure structure on $X^{3}, c_{\text {dis }}$ is the discrete $\mathcal{V}$-valued closure structure on $X^{2} \vee \triangle X^{2}$ and $\pi_{j}: X^{3} \rightarrow X$ is the projection map for $j=1,2,3$.

Let $u \in X^{2} \vee_{\triangle} X^{2}$ and $D$ be a non-empty subset of $X^{2} \vee_{\triangle} X^{2}$, and for all $x, y \in X$ with $x \neq y$, there exist $B \subset X$ with $x \in B, y \notin B$ and $C \subset X$ with $y \in C, x \notin C$ such that $c(B)(y) \wedge k=\perp=c(C)(x) \wedge k$.

Case I: If $\nabla u=(x, x) \in \nabla D$ for some $x \in X$, then $u=(x, x)_{1}$ or $u=(x, x)_{2} \in D$ and consequently, $\bar{c}(D)(u)=k$, where $k$ is the tensor neutral element.

Case II: If $\nabla u=(x, x) \notin \nabla D$, then $c_{\text {dis }}(\nabla D)(\nabla u)=\perp$ since $c_{d i s}$ is the discrete $\mathcal{V}$-valued closure structure and consequently, $\bar{c}(D)(u)=\perp$.

Case III: Suppose $\nabla u=(x, y)$ for some $x, y \in X$ with $x \neq y$ and it follows that $u=(x, y)_{i}, i=1,2$.

(i) If $u=(x, y)_{i} \in D$ for $i=1,2$, then $\nabla u \in \nabla D$ and $\pi_{j} S u \in \pi_{j} S D$ for $j=1,2,3$, and consequently,

$$
\bar{c}(D)(u)=\bigwedge\left\{c_{d i s}(\nabla D)(\nabla u), c\left(\pi_{j} S D\right)\left(\pi_{j} S u\right): j=1,2,3\right\}=k .
$$

(ii) If $u \notin D$, then $\nabla u=(x, y) \notin \nabla D$, and it follows that

$$
c_{d i s}(\nabla D)(\nabla u)=c_{d i s}(\nabla D)(x, y)=\perp,
$$

and consequently, $\bar{c}(D)(u)=\perp$.

(iii) Suppose that $u=(x, y)_{1} \notin D$ but $\left\{(x, y)_{2}\right\} \in D$. It follows that

$$
\begin{gathered}
c_{d i s}(\nabla D)(\nabla u)=c_{d i s}(\nabla D)\left(\nabla(x, y)_{1}\right)=k, \\
k \leq c\left(\pi_{1} S D\right)\left(\pi_{1} S u\right)=c\left(\pi_{1} S D\right)\left(\left(\pi_{1} S(x, y)_{1}\right)=c\left(\pi_{1} S D\right)((x)\right.
\end{gathered}
$$

since $x \in \pi_{1} S D$,

$$
c(B)(y)=c\left(\pi_{2} S D\right)\left(\pi_{2} S u\right)=c\left(\pi_{2} S D\right)\left(\pi_{2} S(x, y)_{1}\right)=c(\{x\})(y)
$$


and

$$
k \leq c\left(\pi_{3} S D\right)\left(\pi_{3} S u\right)=c\left(\pi_{3} S D\right)\left(\pi_{3} S(x, y)_{1}\right)=c\left(\pi_{3} S D\right)(y)
$$

since $y \in \pi_{3} S D$. By Lemma 2.5,

$$
\begin{aligned}
\bar{c}(D)(u)= & \bigwedge\left\{c_{d i s}(\nabla D)\left(\nabla(x, y)_{1}\right), c\left(\pi_{1} D\right)\left(\pi_{1} S(x, y)_{1}\right),\right. \\
& \left.c\left(\pi_{2} S D\right)\left(\pi_{2} S(x, y)_{1}\right), c\left(\pi_{3} S D\right)\left(\pi_{3} S(x, y)_{1}\right)\right\} \\
= & \bigwedge\{k, c(B)(y)\}=\perp
\end{aligned}
$$

since $c(B)(y) \wedge k=\perp$.

(iv) Similarly, if $u=(x, y)_{2} \notin D$ but $(x, y)_{1} \in D$, then

$$
\begin{aligned}
\bar{c}(D)(u)= & \bigwedge\left\{c_{\text {dis }}(\nabla D)\left(\nabla(x, y)_{2}\right), c\left(\pi_{1} D\right)\left(\pi_{1} S(x, y)_{2}\right),\right. \\
& \left.c\left(\pi_{2} S D\right)\left(\pi_{2} S(x, y)_{2}\right), c\left(\pi_{3} S D\right)\left(\pi_{3} S(x, y)_{2}\right)\right\} \\
= & \bigwedge\{k, c(C)(x)\}=\perp
\end{aligned}
$$

since $k \wedge c(C)(x)=\perp$, and consequently, $\bar{c}(D)(u)=\perp$.

Hence, for all $u \in X^{2} \vee \triangle X^{2}$ and all non-empty subset $D$ of $X^{2} \vee_{\triangle} X^{2}$, we have

$$
\bar{c}(D)(u)= \begin{cases}k, & u \in D, \\ \perp, & u \notin D .\end{cases}
$$

By Lemma $2.6(\mathrm{i}), \bar{c}$ is the discrete $\mathcal{V}$-valued closure structure on $X^{2} \vee \triangle X^{2}$. Thus, $(X, c)$ is $T_{1}$.

Corollary 3.10. Let $(X, c)$ be a $\mathcal{V}$-valued closure space, where $\mathcal{V}$ is an integral quantale. $(X, c)$ is $T_{1}$ if and only if for all $x, y \in X$ with $x \neq y$, there exist $B \subseteq X$ with $x \in B$, $y \notin B$ and $C \subseteq X$ with $y \in C, x \notin C$ such that $c(B)(y)=\perp=c(C)(x)$.

Proof. It follows from Theorem 3.9 and the definition of integral quantales.

Example 3.11. Consider $V=[0,1]$ (the real unit interval) with $\leq$ as the partial order, the product . as the quantale operation and 1 as the identity element. Then $\mathcal{V}=(([0,1], \leq$ )$, \cdot, 1)$ is a quantale. Let $X=\{a, b, c\}$ and $c: P(X) \longrightarrow \mathcal{V}^{X}$ be a map defined by for all $x \in X$ and all non-empty subset $A$ of $X$,

$$
(c A)(x)= \begin{cases}1, & x \in A, \\ 1 / 3, & x \notin A .\end{cases}
$$

Clearly, $(X, c)$ is a $\mathcal{V}$-valued closure space. By Theorem $3.8,(X, c)$ is $T_{0}$ but by Theorems 3.6 and $3.9,(X, c)$ is neither $\overline{T_{0}}$ nor $T_{1}$.

\section{Pre-Hausdorff and Hausdorff quantale-valued closure spaces}

Definition 4.1. Let $U: \mathcal{E} \longrightarrow$ Set be a topological functor and $X \in O b(\mathcal{E})$ with $U(X)=$ $B$.

(i) $X$ is called Pre- $\overline{T_{2}}$ provided that the initial lifts of $U$-sources $\left\{A: B^{2} \vee_{\triangle} B^{2} \longrightarrow\right.$ $U\left(X^{3}\right)=B^{3}$ and $\left.S: B^{2} \vee_{\triangle} B^{2} \longrightarrow U\left(X^{3}\right)=B^{3}\right\}$ coincide $[5,10]$.

(ii) $X$ is called $\overline{T_{2}}$ provided that $X$ is $\overline{T_{0}}$ and Pre- $\overline{T_{2}}[5,11]$.

(iii) $X$ is called $N T_{2}$ provided that $X$ is $T_{0}$ and Pre- $\overline{T_{2}}$ [11].

Remark 4.2. In Top (the category of topological spaces and continuous maps), $\overline{T_{2}}$ and $N T_{2}$ are reduced to Hausdorff topological space $(X, \tau)$, i.e., for each $x, y \in X$ with $x \neq y$, there exists a neighborhood $U_{x}$ of $x$ which doesn't contain $y$ and there exists a neighborhood $U_{y}$ of $y$ which doesn't contain $x$ such that $U_{x} \cap U_{y}=\emptyset[11]$. 
Theorem 4.3. Let $(X, c)$ be a $\mathcal{V}$-valued closure space, where $\mathcal{V}$ is an integral quantale. $(X, c)$ is Pre- $\overline{T_{2}}$ if and only if for all $x, y \in X$ with $x \neq y$, there exist $B \subseteq X$ with $x \in B$, $y \notin B$ and $C \subseteq X$ with $y \in C, x \notin C$ such that

$$
c(B)(y) \wedge c(C)(x)=c(B)(y)=c(C)(x) .
$$

Proof. Suppose $(X, c)$ is Pre- $\overline{T_{2}}$. Let $\pi_{j}: X^{3} \longrightarrow X, j=1,2,3$ be the projection map. For all $x, y \in X$ with $x \neq y$, let $u=(x, y)_{1} \in X^{2} \vee \triangle X^{2}$ and $\left\{(x, y)_{2}\right\} \subseteq D \subseteq X^{2} \vee \triangle X^{2}$. Note that

$$
c\left(\pi_{1} A D\right)\left(\pi_{1} A(x, y)_{1}\right)=c\left(\pi_{1} A D\right)(x)=k=\top=c\left(\pi_{1} S D\right)\left(\pi_{1} S(x, y)_{1}\right)
$$

since $x \in \pi_{1} A D$ and $x \in \pi_{1} S D$,

$$
c\left(\pi_{2} A D\right)\left(\pi_{2} A(x, y)_{1}\right)=c\left(\pi_{2} A D\right)(y)
$$

since $y \notin \pi_{2} A D$ and $x \in \pi_{2} A D$,

$$
c\left(\pi_{2} S D\right)\left(\pi_{2} S(x, y)_{1}\right)=c\left(\pi_{2} S D\right)(y)
$$

since $y \notin \pi_{2} S D$ and $x \in \pi_{2} S D$. It follows that

$$
\begin{gathered}
c\left(\pi_{2} A D\right)\left(\pi_{2} A(x, y)_{1}\right)=c\left(\pi_{2} A D\right)(y)=c\left(\pi_{2} S D\right)\left(\pi_{2} S(x, y)_{1}\right)=c(B)(y), \\
c(C)(x)=c\left(\pi_{3} A D\right)\left(\pi_{3} A(x, y)_{1}\right)=c\left(\pi_{3} A D\right)(x)
\end{gathered}
$$

and

$$
c\left(\pi_{3} S D\right)\left(\pi_{3} S(x, y)_{1}\right)=c\left(\pi_{3} S D\right)(y)=k=\top
$$

since $y \in \pi_{3} S D$. This implies

$$
\begin{aligned}
\bigwedge\left\{c\left(\pi_{j} A D\right)\left(\pi_{j} A(x, y)_{1}\right) ; j=1,2,3\right\} & =\bigwedge\left\{c\left(\pi_{1} A D\right)(x), c\left(\pi_{2} A D\right)(y), c\left(\pi_{3} A D\right)(x)\right\} \\
& =\bigwedge\{c(B)(y), c(C)(x)\} .
\end{aligned}
$$

Similarly,

$$
\begin{aligned}
\bigwedge\left\{c\left(\pi_{j} S D\right)\left(\pi_{j} S(x, y)_{1}\right) ; j=1,2,3\right\} & =\bigwedge\left\{c\left(\pi_{1} S D\right)(x), c\left(\pi_{2} S D\right)(y), c\left(\pi_{3} S D\right)(y)\right\} \\
& =c(B)(y) .
\end{aligned}
$$

Since $(X, c)$ is Pre- $\overline{T_{2}}$, we have

$$
\bigwedge\left\{c\left(\pi_{j} A D\right)\left(\pi_{j} A(x, y)_{1}\right) ; j=1,2,3\right\}=\bigwedge\left\{c\left(\pi_{j} S D\right)\left(\pi_{j} S(x, y)_{1}\right) ; j=1,2,3\right\},
$$

and consequently,

$$
\bigwedge\{c(B)(y), c(C)(x)\}=c(B)(y) .
$$

Let $u=(x, y)_{2} \in X^{2} \vee \triangle X^{2}$ and $\left\{(x, y)_{1}\right\} \subseteq D \subseteq X^{2} \vee_{\triangle} X^{2}$. By a similar verification, we have $\bigwedge\{c(B)(y), c(C)(x)\}=c(C)(x)$ and consequently,

$$
\bigwedge\{c(B)(y), c(C)(x)\}=c(C)(x)=c(B)(y) .
$$

Conversely, let $\bar{c}_{A}$ and $\bar{c}_{S}$ be the two initial $\mathcal{V}$-valued closure structures on $X^{2} \vee \triangle X^{2}$ induced by $A: X^{2} \vee_{\triangle} X^{2} \longrightarrow U\left(X^{3}, c^{3}\right)=X^{3}$ and $S: X^{2} \vee_{\triangle} X^{2} \longrightarrow U\left(X^{3}, c^{3}\right)=X^{3}$ respectively, where $c^{3}$ is the product $\mathcal{V}$-valued closure structure on $X^{3}$ induced by the projection map $\pi_{j}: X^{3} \longrightarrow X$ for $j=1,2,3$. We need to show that for all $u \in X^{2} \vee_{\triangle} X^{2}$ and all non-empty subset $D$ of $X^{2} \vee_{\triangle} X^{2}, \bar{c}_{A}(D)(u)=\bar{c}_{S}(D)(u)$.

Case (I): If $u \in D$, then $\bar{c}_{A}(D)(u)=\bar{c}_{S}(D)(u)$ since $\bar{c}_{A}(D)(u)=\bar{c}_{S}(D)(u)=k=\top$.

Case (II): Suppose $u \notin D$ and they are in the same component of $X^{2} \vee_{\triangle} X^{2}$. This implies that $u=(x, y)_{i}$, and $\left\{(z, w)_{i}\right\} \subseteq D$ for $i=1,2$, where $x, y, z, w \in X$. For $i=1$, we have

$$
\begin{gathered}
c\left(\pi_{1} A D\right)\left(\pi_{1} A u\right)=c\left(\pi_{1} A D\right)(x), \\
c\left(\pi_{2} A D\right)\left(\pi_{2} A u\right)=c\left(\pi_{2} A D\right)\left(\pi_{2} A(x, y)_{1}\right)=c\left(\pi_{2} A D\right)(y),
\end{gathered}
$$


and

$$
c\left(\pi_{3} A D\right)\left(\pi_{3} A u\right)=c\left(\pi_{3} A D\right)\left(\pi_{3} A(x, y)_{1}\right)=c\left(\pi_{3} A D\right)(x) .
$$

Note that

$$
\begin{aligned}
\bar{c}_{A}(A D)(A u)= & \bigwedge\left\{c\left(\pi_{j} A D\right)\left(\pi_{j} A u\right): j=1,2,3\right\} \\
= & \bigwedge\left\{c\left(\pi_{1} A D\right)\left(\pi_{1} A(x, y)_{1}\right), c\left(\pi_{2} A D\right)\left(\pi_{2} A(x, y)_{1}\right),\right. \\
& \left.c\left(\pi_{3} A D\right)\left(\pi_{3} A(x, y)_{1}\right)\right\} \\
= & \bigwedge\left\{c\left(\pi_{1} A D\right)(x), c\left(\pi_{2} A D\right)(y)\right\} .
\end{aligned}
$$

and

$$
\begin{aligned}
\bar{c}_{S}(S D)(S u) & =\bigwedge\left\{c\left(\pi_{j} S D\right)\left(\pi_{j} S u\right): j=1,2,3\right\} \\
& =\bigwedge\left\{c\left(\pi_{1} S D\right)(x), c\left(\pi_{2} S D\right)(y)\right\} .
\end{aligned}
$$

This implies $\bar{c}_{A}(A D)(A u)=\bar{c}_{S}(S D)(S u)$.

For $i=2$, it follows that

$$
\bar{c}_{A}(A D)(A u)=\bar{c}_{A}(A D)\left(A(x, y)_{2}\right)=\bar{c}_{S}(S D)\left(S(x, y)_{2}\right)=\bar{c}_{S}(S D)(S u) .
$$

Case (III): Suppose $u \notin D$ and they are in the different components of $X^{2} \vee_{\triangle} X^{2}$. We have the following cases.

(a) If $u=(x, y)_{1}$ or $(y, x)_{1}$ and $\left\{(x, y)_{2}\right\} \subseteq D$ or $\left\{(y, x)_{2}\right\} \subseteq D$ for all $x \neq y$.

Suppose $u=(x, y)_{1}$ and $\left\{(x, y)_{2}\right\} \subseteq D$ (resp. $\left.\left\{(y, x)_{2}\right\} \subseteq D\right)$. Then by Remark 2.5 , it follows that

$$
\begin{aligned}
\bar{c}_{A}(A D)(A u) & =\bigwedge\left\{C\left(\pi_{j} A D\right)\left(\pi_{j} A u\right): j=1,2,3\right\} \\
& =\bigwedge\left\{c\left(\pi_{1} A D\right)\left(\pi_{1} A(x, y)_{1}\right), c\left(\pi_{2} A D\right)\left(\pi_{2} A(x, y)_{1}\right), c\left(\pi_{3} A D\right)\left(\pi_{3} A(x, y)_{1}\right)\right\} \\
& =\bigwedge\left\{c\left(\pi_{2} A D\right)(y), c\left(\pi_{3} A D\right)(x), \top\right\} \\
& =c(B)(y) \wedge c(C)(x) \quad(\operatorname{resp} . c(B)(y))
\end{aligned}
$$

and

$$
\begin{aligned}
\bar{c}_{S}(S D)(S u) & =\bigwedge\left\{C\left(\pi_{j} S D\right)\left(\pi_{j} S u\right): j=1,2,3\right\} \\
& =\bigwedge\left\{c\left(\pi_{1} S D\right)\left(\pi_{1} S(x, y)_{1}\right), c\left(\pi_{2} S D\right)\left(\pi_{2} S(x, y)_{1}\right),\left(\pi_{3} S D\right)\left(\pi_{3} S(x, y)_{1}\right)\right\} \\
& =\bigwedge\{\top, c(B)(y)\} \\
& =c(B)(y) \quad(\text { resp. } c(B)(y) \wedge c(C)(x)) .
\end{aligned}
$$

By the assumption, we have $\bar{c}_{A}(A D)(A u)=\bar{c}_{S}(S D)(S u)$.

Similarly, if $u=(y, x)_{1}$ and $\left\{(x, y)_{2}\right\} \subseteq D$ or $\left\{(y, x)_{2}\right\} \subseteq D$ for all $x \neq y$. It follows that $\bar{c}_{A}(A D)(A u)=\bar{c}_{S}(S D)(S u)$.

(b) If $u=(x, y)_{2}$ or $(y, x)_{2}$ and $\left\{(x, y)_{1}\right\} \subseteq D$ or $\left\{(y, x)_{1}\right\} \subseteq D$ for all $x \neq y$.

Let $u=(x, y)_{2}$ and $\left\{(x, y)_{1}\right\} \subseteq D,\left(\right.$ resp. $\left.\left\{(y, x)_{1}\right\} \subseteq D\right)$. Then it follows from Remark 2.5 that

$$
\begin{aligned}
\bar{c}_{A}(A D)(A u) & =\bigwedge\left\{C\left(\pi_{j} A D\right)\left(\pi_{j} A u\right): j=1,2,3\right\} \\
& =\bigwedge\left\{c\left(\pi_{1} A D\right)\left(\pi_{1} A(x, y)_{2}\right), c\left(\pi_{2} A D\right)\left(\pi_{2} A(x, y)_{2}\right), c\left(\pi_{3} A D\right)\left(\pi_{3} A(x, y)_{2}\right)\right\} \\
& =\bigwedge\left\{\top, c\left(\pi_{2} A D\right)(x), c\left(\pi_{3} A D\right)(y)\right\} \\
& =c(B)(y) \wedge c(C)(x) \quad(\operatorname{resp} . c(C)(x))
\end{aligned}
$$


and

$$
\begin{aligned}
\bar{c}_{S}(S D)(S u) & =\bigwedge\left\{C\left(\pi_{j} S D\right)\left(\pi_{j} S u\right): j=1,2,3\right\} \\
& =\bigwedge\left\{c\left(\pi_{1} S D\right)\left(\pi_{1} S(x, y)_{2}\right), c\left(\pi_{2} S D\right)\left(\pi_{2} S(x, y)_{2}\right), c\left(\pi_{3} S D\right)\left(\pi_{3} S(x, y)_{2}\right)\right\} \\
& =\bigwedge\{\top, c(C)(x)\} \\
& =c(C)(x) \quad(\text { resp. } c(B)(y) \wedge c(C)(x)) .
\end{aligned}
$$

Similarly, if $u=(y, x)_{2}$ and $\left\{(x, y)_{1}\right\} \subseteq D$ or $\left\{(y, x)_{1}\right\} \subseteq D$ for all $x \neq y$, then it follows that $\bar{c}_{A}(A D)(A u)=\bar{c}_{S}(S D)(S u)$.

(c) For any three (resp. four) distinct points $x, y, z$ (resp. $w) \in X$, similar to above cases, $\bar{c}_{A}(A D)(A u)=\bar{c}_{S}(S D)(S u)$.

Therefore, for all $u \in X^{2} \vee_{\triangle} X^{2}$ and all non-empty subset $D$ of $X^{2} \vee_{\triangle} X^{2}, \bar{c}_{A}(D)(u)=$ $\bar{c}_{S}(D)(u)$. Thus, $(X, c)$ is Pre- $\overline{T_{2}}$.

Theorem 4.4. Let $\mathcal{V}$ be an integral quantale, and let $(X, c)$ be a $\mathcal{V}$-valued closure space. $(X, c)$ is $\overline{T_{2}}$ if and only if $(X, c)$ is a discrete $\mathcal{V}$-valued closure space.

Proof. It follows from the definition of integral quantales, Definition 4.1 (ii), Lemma 2.6 (i) and Theorems 3.6 and 4.3 .

Theorem 4.5. Let $\mathcal{V}$ be an integral quantale, and let $(X, c)$ be a $\mathcal{V}$-valued closure space. The followings are equivalent.

(i) $(X, c)$ is $T_{1}$.

(ii) $(X, c)$ is $\overline{T_{2}}$.

(iii) $(X, c)$ is a discrete $\mathcal{V}$-valued closure space.

Proof. The proof follows from Lemma 2.6 (i), and Theorems 3.9 and 4.4.

Theorem 4.6. Let $\mathcal{V}$ be an integral quantale, and let $(X, c)$ be a $\mathcal{V}$-valued closure space. $(X, c)$ is $N T_{2}$ if and only if there exist $x, y \in X$ with $x \neq y$,

$$
c(\{y\})(x)=c(\{x\})(y)<\top .
$$

Proof. It follows from Definition 4.1 (iii) and Theorems 3.8 and 4.3.

Remark 4.7. (I) For any arbitrary topological category, there is no relation between $T_{0}$ and $\overline{T_{0}}$, and between $\overline{T_{2}}$ and $N T_{2}$. For example,

(a) In category Cls of closure spaces and continuous maps, Pre- $\overline{T_{2}}=N T_{2}=$ $\overline{T_{2}} \Rightarrow T_{1}=\overline{T_{0}} \Rightarrow T_{0}[12]$.

(b) In category $\mathbf{C H Y}$ of of Cauchy spaces and Cauchy continuous maps, $T_{0}=$ $\overline{T_{0}}=T_{1}=\overline{T_{2}} \Longrightarrow$ Pre- $\overline{T_{2}}$ [22].

(c) In ConFCO (the category of constant filter convergence spaces and continuous maps), $\overline{T_{2}}=N T_{2} \Rightarrow T_{0}=\overline{T_{0}}=T_{1}$ but in ConLFCO (the category of constant local filter convergence spaces and continuous maps), $T_{0} \Longrightarrow \overline{T_{0}}=T_{1}$ and $T_{0}=N T_{2} \Longrightarrow \overline{T_{2}}[6]$.

(d) In L-App (category of $L$-gauge space (resp. $L$-distance approach space) and contraction maps) [20], local $T_{1}$, i.e., $T_{1}$ at $p$ implies local $\overline{T_{0}}$, i.e., $\overline{T_{0}}$ at $p$ [36].

(II) In V-Cls with $\mathcal{V}$ as an integral quantale, by Theorems 3.6-3.9 and 4.5, $\overline{T_{2}}=T_{1} \Longrightarrow$ $\overline{T_{0}} \Longrightarrow T_{0}$ but converse is not true in general by Example 3.11. Moreover, by Theorems 4.3-4.6, if $(X, c)$ is $\overline{T_{2}}$, then it is Pre- $\overline{T_{2}}$ and $N T_{2}$.

Acknowledgment. We would like to thank the referees for their valuable and helpful suggestions that improved the paper radically. This work is supported by the National Natural Science Foundations of China (12071033 and 11701122). 


\section{References}

[1] J. Adamek, H. Herrlich, and G.E. Strecker, Abstract and Concrete Categories, John Wiley \& Sons, New York, 1990.

[2] D. Aerts, Foundations of quantum physics: a general realistic and operational approach, Int. J. Theoret. Phys. 38 (1), 289-358, 1999.

[3] D. Aerts, E. Colebunders, A. Van der Voorde and B. Van Steirteghem, State property systems and closure spaces: a study of categorical equivalence, Int. J. Theoret. Phys. 38 (1), 359-385, 1999.

[4] G. Aumann, Kontaktrelationen, Bayer. Akad. Wiss. Math.-Nat. Kl. Sitzungsber, 6777, 1970.

[5] M. Baran, Separation Properties, Indian J. Pure Appl. Math. 23, 333-341, 1991.

[6] M. Baran, Separation properties in the categories of Constant Convergence Spaces, Turkish J. Math. 18, 238-248, 1994.

[7] M. Baran, Separation Properties in Topological Categories, Math. Balkanica. 10, 3948, 1996.

[8] M. Baran, $T_{3}$ and $T_{4}$-objects in topological categories, Indian J. Pure Appl. Math. 29, 59-70, 1998.

[9] M. Baran, Completely regular objects and normal objects in topological categories, Acta Math. Hungar. 80 (3), 211-224, 1998.

[10] M. Baran, Pre $T_{2}$ objects in topological categories, Appl. Categ. Structures, 17, 591602, 2009.

[11] M. Baran and H. Altındis, $T_{2}$ objects in topological categories, Acta Math. Hungar. 71 (1-2), 41-48, 1996.

[12] M. Baran, D. Tokat and M. Kula, Connectedness and Separation in the Category of Closure Spaces, Filomat 24 (2), 67-79, 2010.

[13] G. Birkhoff, Lattice Theory, American Mathematical Society, Providence, Rhonde Island, 1940.

[14] E. Čech, On bicompact spaces, Ann. Math. 38, 823-844, 1937.

[15] D. Deses, E. Giuli and E. Lowen-Colebunders, On the complete objects in the category of $T_{0}$ closure spaces, Appl. Gen. Topology, 4, 25-34, 2003.

[16] M. Erné, Lattice representations for categories of closure spaces, Categorical Topology, Sigma Series in Pure Mathematics 5, Heldermann Verlag Berlin, 197-222, 1984.

[17] R.C. Flagg, Quantales and continuity spaces, Algebra Univers. 37, 257-276, 1997.

[18] P. Hertz, Über Axiomensysteme für beliebige Satzsysteme, Teil I, Math. Ann. 87, 246-269, 1922.

[19] G. Jäger, Probabilistic Approach Spaces, Math. Bohem. 142 (3), 277-298, 2017.

[20] G. Jäger and W. Yao, Quantale-valued gauge spaces, Iran. J. Fuzzy Syst. 15 (1), 103-122, 2018.

[21] P.T. Johnstone, Stone Spaces, L. M. S. Mathematics Monograph: No. 10. Academic Press, New York, 1977.

[22] M. Kula, A note on Cauchy spaces, Acta Math. Hungar. 133 (1-2), 14-32, 2011.

[23] K. Kuratowski, Sur L'operation $\bar{A}$ de l'Analysis Situs, Fund. Math. 3, 182-199, 1992.

[24] H. Lai and W. Tholen, Quantale-valued topological spaces via closure and convergence, Topology Appl. 30, 599-620, 2017.

[25] H. Lai and W. Tholen, A note on the Topologicity of Quantale-valued Topological spaces, Log. Methods Comput. Sci. 13 (3:12), 1-13, 2017.

[26] R. Lowen, Approach spaces: The missing link in the Topology-Uniformity-Metric triad, Oxford University Press, 1997.

[27] M.V. Mielke, Separation axioms and geometric realizations, Indian J. Pure Appl. Math. 25, 711-722, 1994. 
[28] M.V. Mielke, Hausdorff separation and decidability, in: Symposium on Categorical Topology, University of Cape Town, Rondebosch, 155-160, 1999.

[29] E.H. Moore, On a form of general analysis with applications to linear differential and integral equations, in: Atti del IV Congress. Internationale di Mat. II, Roma, 98-114, 1909.

[30] B. Pang, Categorical properties of L-fuzzifying convergence spaces, Filomat 32 (11), 4021-4036, 2018.

[31] B. Pang, Convenient properties of stratified L-convergence tower spaces, Filomat 33 (15), 4811-4825, 2019.

[32] B. Pang, Hull operators and interval operators in the $(L, M)$-fuzzy convex spaces, Fuzzy Sets and Systems 405, 106-127, 2021.

[33] B. Pang and F.-G. Shi, Strong inclusion orders between L-subsets and its applications in L-convex spaces, Quaest. Math. 41 (8), 1021-1043, 2018.

[34] R.S. Pierce, Closure spaces with applications to ring theory, in: Lectures on Rings and Modules, Lecture Notes in Mathematics 246, Springer, Berlin, Heidelberg, 1972.

[35] G. Preuss, Foundations of topology: an approach to convenient topology, Kluwer Academic Publishers, Dordrecht, 2002.

[36] M. Qasim and S. Özkan, The notions of closedness and D-connectedness in Quantalevalued Approach Spaces, Categ. Gen. Alg. Struct. Appl. 12, 149-173, 2020.

[37] G.J. Seal, Canonical and op-canonical lax algebras, Theory Appl. Categ. 14 (10), 221-243, 2005.

[38] S. Weck-Schwarz, $T_{0}$-objects and separated objects in topological categories, Quaest. Math. 14 (3), 315-325, 1991.

[39] Z.-Y. Xiu and Q.-H. Li, Degrees of L-continuity for mappings between L-topological spaces, Mathematics 7 (11), 1013-1028, 2019.

[40] Z.-Y. Xiu, Q.-H. Li and B. Pang, Fuzzy convergence structures in the framework of L-convex spaces, Iran. J. Fuzzy Syst. 17 (4), 139-150, 2020. 